This is the postprint version of the following article: Mejias SH, Couleaud P, Casado S, et al. Assembly of designed protein scaffolds into monolayers for nanoparticle patterning. Colloids and Surfaces B: Biointerfaces. 2016;141:93-101. doi: 10.1016/j.colsurfb.2016.01.039. This article may be used for non-commercial purposes in accordance with Elsevier Terms and Conditions for Self-Archiving. 


\section{Assembly of designed protein scaffolds into monolayers for nanoparticle patterning}

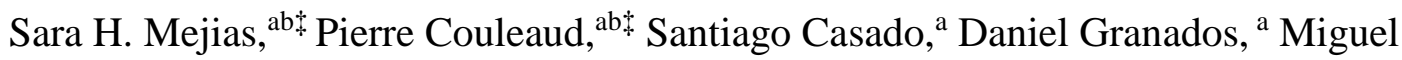
Angel Garcia ${ }^{\text {ac }}$ Jose M. Abad ${ }^{\text {ad* }}$ and Aitziber L. Cortajarena. ${ }^{\text {ab* }}$

a IMDEA-Nanociencia, Universidad Autónoma de Madrid,Cantoblanco, 28049 Madrid, Spain; Tel: 34 912998857; E-mail: aitziber.lopezcortajarena@imdea.org

${ }^{b}$ Centro Nacional de Biotecnología (CNB-CSIC) - IMDEA Nanociencia Associated Unit, Universidad Autónoma de Madrid, Cantoblanco, 28049 Madrid, Spain.

c Instituto de Cerámica y Vidrio (ICV-CSIC), Cantoblanco, 28049 Madrid, Spain.

${ }^{d}$ Departamento de Química Analítica y Análisis Instrumental, Facultad de Ciencias, Universidad Autónoma de Madrid, 28049 Madrid, Spain. E-mail: josemaria.abad@uam.es

* These authors contributed equally to this work.

Total words: $\mathbf{5 2 3 1}$

Number of figures: 6 


\title{
Assembly of designed protein scaffolds into monolayers for nanoparticle patterning
}

\author{
Sara H. Mejias, ${ }^{\text {ab } \sharp}$ Pierre Couleaud, ${ }^{\text {ab }}$ Santiago Casado, ${ }^{\mathrm{a}}$ Daniel Granados, ${ }^{\mathrm{a}}$ Miguel \\ Angel Garcia ${ }^{\text {ac }}$ Jose M. Abad ${ }^{\text {ad* }}$ and Aitziber L. Cortajarena. ${ }^{\text {ab* }}$ \\ a IMDEA-Nanociencia, Universidad Autónoma de Madrid ,Cantoblanco, 28049 Madrid, Spain; Tel: 34 \\ 912998857; E-mail: aitziber.lopezcortajarena@imdea.org \\ ${ }^{b}$ Centro Nacional de Biotecnología (CNB-CSIC) - IMDEA Nanociencia Associated Unit, Universidad \\ Autónoma de Madrid, Cantoblanco, 28049 Madrid, Spain. \\ ${ }^{c}$ Instituto de Cerámica y Vidrio (ICV-CSIC), Cantoblanco, 28049 Madrid, Spain. \\ ${ }^{d}$ Departamento de Química Analítica y Análisis Instrumental, Facultad de Ciencias, Universidad Autónoma de \\ Madrid, 28049 Madrid, Spain. E-mail: josemaria.abad@uam.es \\ * These authors contributed equally to this work.
}

\section{Abstract}

The controlled assembly of building blocks to achieve new nanostructured materials with defined properties at different length scales through rational design is the basis and future of bottom-up nanofabrication. This work describes the assembly of the idealized protein building block, the consensus tetratricopeptide repeat (CTPR), into monolayers by oriented immobilization of the blocks. The selectivity of thiol-gold interaction for an oriented immobilization has been verified by comparing a non-thiolated protein building block. The physical properties of the CTPR protein thin biomolecular films including topography, thickness, and viscoelasticity, are characterized. Finally, the ability of these scaffolds to act as templates for inorganic nanostructures has been demonstrated by the formation of wellpacked gold nanoparticles (GNPs) monolayer patterned by the CTPR monolayer.

\section{Keywords}

repeat protein; protein design; self-assembly; monolayer; gold nanoparticles; biomolecular templating; building blocks, nanoparticle 2D arrays, nanopatterning. 


\section{Introduction}

The use of building blocks and self-assembly is acknowledged as a versatile strategy for the fabrication of multifunctional nanostructures. Inorganic building blocks and nanoparticles have already shown great potential in the fabrication of new materials and multifunctional structures.[1-4] Additionally, the synthesis of hybrid organic-inorganic multifunctional nanostructures has also been developed.[5, 6] At the contrary, the use of purely organic building blocks, such as biomolecules, has not yet been fully exploited in nanotechnology. Self-assembly of biological molecules into defined functional structures has tremendous potential in nanopatterning and in the design of novel materials and functional devices.[7-15] Moreover, templating inorganic nanostructures, such as gold nanoparticles, using biomolecules is a challenging way to generate ordered inorganic materials[16, 17] for example to produce memory devices, non-linear optical and sensing devices, and nanowires.[18, 19] These strategies permit to have a high control at the nanometer scale, that is the major limitation of the conventional lithographic top-down processes used actually.

Molecular self-assembly is a process by which complex three-dimensional structures with specified functions are constructed from simple molecular building blocks. These methodologies enable the precise control over the structure but also over the function that can be encoded in the biomolecules. In order to take advantage of the great potential of using biomolecules to generate functional templates, it is key to have a molecular understanding of the building blocks and a control over interactions that drive their assembly. Additionally, green, easy and innovative strategies to have a precise control at the atomic scale for the development of scaffold and patterned structures are still to be found.

In that sense the use of proteins is of great interest as a new strategy for bottom-up approaches in nanotechnology.[20] For example, compared to other approaches used for patterning nanoparticles, such as nanolithography, any bottom up approach is more cost effective and easier to implement. In this sense, interfacial bottom up 2D assembly of nanoparticles has become a novel and widely used strategy, however it presents some limitations, including the difficulty of assembling nanoparticles with different coatings and mostly the low adhesion of the film when transferred to the substrate. [21] Nanoparticle templating by proteins combines the cost efficiency of bottom-up approaches with the capability of covalent bioconjugation with sub-nanometric resolution. The complexity and sophistication of protein-based structures in nature hints to the great potential of designed protein-based templating. In particular, the use of designed repeat proteins as the building 
blocks for fabrication and patterning provides several advantages.[22] Repeat proteins present a modular structure defined by local repeated interactions and are composed of tandem arrays of the same small structural motif.[23, 24] Their simple architecture makes easier to understand the basic rules that relate sequence to structure for these repeated modules and make them ideal molecular building blocks.[25, 26]

Consensus tetratricopeptide repeat (CTPR)[23] is an idealized 34 amino acid helix-turnhelix repeat module that can be combined in tandem to form CTPR proteins with different numbers of repeats from 2 to 20 so called CTPR2 to CTPR20 proteins.[27] CTPR proteins display superhelical structures, in which eight repeats comprise one full turn of the superhelix (Fig. 1A).[27, 28] Additionally, the stability and ligand binding properties of CTPRs can be modulated in a predictable manner, which opens the opportunity to tune the building block properties for specific applications.[29-32] We have recently used these modules to show the specific functionalization of patterned polymeric surfaces.[33, 34] Moreover, it has been shown that under certain conditions CTPR proteins self-assemble into ordered structures including ordered protein films comprised,[35] and linear nanofibers in solution [36, 37], mimicking the packing observed in the crystal forms of CTPRs.[27, 28, 38] These results illustrate the potential of these protein modules as self-assembling building blocks.

One key component for the assembly is the side-to-side lateral interactions between CTPR superhelices.[35, 39] Fig. 1A schematically shows the potential alignment of CTPR superhelices. In this work, we focus on the self-assembly of the CTPR20-Cys protein composed of 20 identical CTPR repeats with a single C-terminal cysteine. CTPR20 is chosen since it is the longest CTPR that can be produced recombinantly with high yield. The longer the CTPR protein the higher the aspect ratio,[26] thus CTPR20 is expected to have higher propensity to be oriented on the surface and form more stable side-to-side interactions. In addition, longer CTPRs show higher stability[40] which is important for future applications. CTPR20-Cys is immobilized on a gold surface through gold-sulfur bond and, driven by the lateral inter-molecular interactions, can generate ordered and oriented protein self-assembled monolayers (SAMs) (Fig. 1B). To characterize the protein's specific adsorption on gold surface and its self-packing, we monitored the immobilization by different techniques including quartz crystal microbalance (QCM), surface plasmon resonance (SPR) and atomic force microscopy (AFM).

Finally, to demonstrate the potential of the repeat protein scaffolds to act as templates for nanoscale patterning, gold nanoparticles (GNPs) were grafted onto immobilized CTPR20Cys through the formation of a covalent amide bond between the terminal amine of the 
protein and the carboxylate groups of the GNPs (Fig. 1C). The resulting functionalized surface with a monolayer of GNPs was characterized by scanning electron microscopy (SEM) providing a final evidence of the oriented monolayer formation of CTPR20-Cys protein and a potential application in the controlled patterning of GNPs (Fig. 1D). The use of GNPs in an ordered monolayer organization is of great interest in field of plasmonics [41] and organic solar cells progress[42] or for the development of localized surface plasmon resonance-based sensor.[43] 


\section{Results and discussion}

The main goal of this work is to demonstrate that repeat proteins can be used as scaffolds for the patterning of inorganic nanostructures, such as gold nanoparticles.

The first step of the study is the adsorption of CTPR20-Cys onto gold surface. Thus, we first compared the immobilization on gold surface of two proteins: CTPR20-Cys for oriented thiol mediated immobilization and CTPR20 without Cys as a non-oriented deposition control. The process was monitored by quartz crystal microbalance (QCM), which is a powerful tool to study interfacial process at surfaces. After stabilization of the temperature, frequency and resistance, purified proteins solutions prepared as previously reported [13, 44] were flowed through the cell. Fig. 2A and 2B present the resulting frequency and resistance changes as a function of time after protein injection. As it can be seen in the Fig. 2A, upon flowing CTPR20-Cys protein, a rapid decrease in the resonant frequency was observed during the first 2 minutes as a result of the protein binding to the surface by chemisorption of cysteine on gold. Afterward, the frequency decreased more slowly until a steady state was reached. Simultaneously with the frequency decrease, an increase in motional resistance was also observed (Fig. 2B). It indicates that the binding of the protein does not result in a rigid load but viscoelastic causing an energy loss by dissipation from the QCM sensor.

The ratio between the motional resistance change and the frequency shift can be used to evaluate the relative influence of viscoelastic and mass loading effects (see below). The shape of the frequency-time profile can be employed to study the kinetics of adsorption by fitting to a first-order kinetics equation (Fig. 2A and Materials and methods section). The values of $\Delta F_{\max }$ and $k$ obtained from the data fit were $-73.2 \mathrm{~Hz}$ and $0.57 \mathrm{~min}^{-1}$, respectively. From the $\Delta F_{\max }$ at the equilibrium, assuming that the frequency decrease is only due to the change in mass arising from the adsorption of the protein, the mass of CTPR20-Cys immobilized on the gold electrode surface was estimated to be $1285 \mathrm{ng} \cdot \mathrm{cm}^{-2}$ using Equation 1. This value corresponds to a surface coverage of about $15.2 \times 10^{-12} \mathrm{~mol} \cdot \mathrm{cm}^{-2}$ considering a molecular mass of 85242 Da for the CTPR20-Cys $\left(9.0 \times 10^{12}\right.$ CTPR20-Cys molecules $\left.\cdot \mathrm{cm}^{-2}\right)$. This value is in agreement with the number of molecules for a compact monolayer of oriented CTPR20Cys proteins, considering a projected area of about $11 \mathrm{~nm}^{2}$ per molecule based on the dimensions from the crystal structure.[27] Additional support to this fact is provided by the comparison with the extensively studied monolayers of n-alkanethiolates onto gold surface $[45,46]$ which result in a maximum coverage of $\sim 4.2 \times 10^{14}$ molecules $\cdot \mathrm{cm}^{-2}$ for molecules that cover $0.217 \mathrm{~nm}^{2}$.[47] For an equally packed CTPR20-Cys molecule $\left(11 \mathrm{~nm}^{2}\right)$ the 
theoretical maximum coverage would be reached with 50 times less molecules $8.4 \times 10^{12}$ molecules $\cdot \mathrm{cm}^{-2}$, which is in the same range of the experimental value of $9.0 \times 10^{12}$ molecules $\cdot \mathrm{cm}^{-2}$.

These results show that the protein units assemble in a compact manner on the surface by thiol chemisorption of cysteine residues onto the gold surface. As it is the case for more simple thiol derivatives, CTPR20-Cys proteins are not randomly deposited since the amount of protein per surface area would be otherwise smaller. To serve as comparison, QCM adsorption measurements of CTPR20 without cysteine were also carried out (Electronic supplementary material). As shown in Fig. S1 flowing CTPR20 gives a final surface coverage of $6.5 \times 10^{-12} \mathrm{~mol} \cdot \mathrm{cm}^{-2}$, significantly lower than for CTPR20-Cys. This result can be associated to the fact that direct adsorption of CTPR20 proceeds through unspecific multipoint attachment of the protein to the surface contrary to the oriented thiol-derivative protein that is tightly adsorbed on the gold surface by an unique single-point interaction.[48, 49] This fact was also put in evidence by studying the thermodynamics of adsorption. The calculated equilibrium proteins coverages from $\Delta$ Fmax for different protein concentrations are fitted to a Langmuir adsorption isotherm (Materials and methods section, Eq. 4) to calculate the saturation protein coverage $\left(\Gamma \mathrm{s}=15.2 \times 10^{-12} \mathrm{~mol} \cdot \mathrm{cm}^{-2}\right)$ (Fig. 2C). In contrast, for CTPR20 without cysteine the $\Gamma$ s was $7.3 \times 10-12 \mathrm{~mol} \cdot \mathrm{cm}^{-2}$ (Fig. S2). The coverage at saturation for CTPR20 is half of the one obtained for CTPR20-Cys, presumably due to a random immobilization of CTPR20 in different orientations.

In order to confirm the validity of conversion the change in frequency to molecular mass from QCM data using Sauerbrey's equation, surface plasmon resonance (SPR) experiments were conducted, as detailed in the Materials \& Methods section, [50, 51] to quantify the amount of protein bound. Fig. 3A shows a SPR experiment carried out employing a CTPR20-Cys protein concentration of $2.5 \mu \mathrm{M}$ to saturate the surface. The mass measured by SPR from the resonance angle shift upon protein binding to the gold surface was approximately $1000 \mathrm{ng} \cdot \mathrm{cm}^{-2}$. This mass is slightly lower than the value obtained from QCM $\left(1285 \mathrm{ng} \cdot \mathrm{cm}^{-2}\right)$, probably due to the uncertainty of the refractive index of the adsorbed protein and to the effect of the hydration water in the QCM measurements. The SPR mass can be considered comparable to the QCM mass confirming that the frequency changes observed in the QCM experiments are mainly ascribed to mass changes as consequence of the protein adsorption since viscoelastic effects should induce a bigger difference between $\triangle m_{\mathrm{QCM}}$ and $\Delta m_{\mathrm{SPR}}$, as previously reported.[52] 
We used AFM to image the CTPR20-Cys proteins immobilized onto gold surfaces. Single CTPR20-Cys molecules were imaged first at a low protein concentration $(10 \mathrm{nM})$ to characterize the features of the individual monomers (Fig. 4A). A single size distribution peak was obtained for individual CTPR20-Cys molecules with an average height of approximately $8 \mathrm{~nm}$, indicating that each bright dot on the image corresponds to a protein monomer (Fig. 4A). The width measurements are not reliable due to the tip convolution.[53] The end to end distance of the CTPR20 superhelix calculated from the crystal structure is approximately 19 nm (Fig. 1A).[27] There are several reasons that will account for the smaller size measured by AFM: (1) CTPR20-Cys adsorption onto gold surface through its thiol group might be tilted with an angle relative to an orthogonal orientation to the surface; (2) vertical elastic tip compression of the elongated superhelical structure, as it has been described for other repeat proteins;[54] (3) AFM characterizes single protein molecules in solution, which might have some flexibility[55] compared to the dimensions determined from the rigid crystal structure of the protein.[56]

Following, CTPR20-Cys monolayer assembly on gold surface was studied. CTPR20Cys at $2.5 \mu \mathrm{M}$ concentration, which guarantees full coverage of the surface according to the equilibrium immobilization studies by QCM (Fig. 2C), was deposited onto annealed gold surfaces. The AFM image shows a densely packed array of protein particles that completely covers the gold surface (Fig. 4B). Under the solution deposition conditions and in the range of protein concentration used $(10 \mathrm{nM}-2.5 \mu \mathrm{M})$, CTPR20-Cys protein is stable, folded, and fully monomeric $[27,40]$, thus is not expected the deposition of multimeric particles. The AFM images at saturation provide insights about the tight packing of the protein molecules on the monolayer and clearly show a full coverage of the surface (Fig. 4B), corroborating a higher occupancy than expected from simple random distribution of individual CTPR20 monomers. The overall roughness analysis of the surface resulted in a RMS roughness of $0.86 \mathrm{~nm}$ with an average height of $3.42 \mathrm{~nm}$, smaller than the one observed for individual CTPR20-Cys proteins (RMS roughness of $1.88 \mathrm{~nm}$ and average size of $6.36 \mathrm{~nm}$ ), in agreement with a compaction of the molecules. The CTPR20-Cys covered surface shown in figure 4B was incubated an excess of dithiothreitol (DTT) that will displace the previously adsorbed CTPR20-Cys, in order to study the specificity of the interaction between CTPR20-Cys and the gold surface. After the incubation and wash of the surface the AFM image (Fig. 4C) shows some dots corresponding 
to individual protein molecules, while most of the protein has been detached from the surface. This result confirms the specificity of the thiol-gold immobilization of the CTPR20-Cys.

In order to determine the thickness of the monolayer imaged by AFM, profilometry experiments were performed on a CTPR20-Cys layer deposited on one half of a gold surface using $2.5 \mu \mathrm{M}$ protein concentration. Three protein layers were measured to give a thickness value of $17.93 \pm 1.05 \mathrm{~nm}$ (Fig.3B), consistent with the formation of a single CTPR20-Cys protein monolayer.

In addition, the ratio between the motional resistance $(\Delta R)$ and the frequency $(\Delta F)$ provides information about the viscoelastic properties of the deposited protein layer.[57] In Fig. 5A, the $\Delta R$ during the course of CTPR20-Cys protein assembly is plotted against $\Delta F$. Two separate regions are observed with different $\Delta R / \Delta F$ slopes indicating that surface coverage has a strong influence on the viscoelastic properties of the protein film. The first region ( $\Delta F$ from 0 to -20 ) shows a linear relationship between $\Delta R / \Delta F$ presenting a steeper slope. It corresponds to the beginning of the adsorption process associated to lower protein densities and therefore less lateral interactions between protein molecules. The deposit tends to be more flexible and more dissipative at this stage. When higher protein densities are reached ( $\Delta F$ from -20 to -60 ), protein molecules can establish lateral interactions with neighbouring molecules, yielding finally to a more rigid and less dissipative film. The change in slope between two regions indicates the transition from individual proteins to a compact state. The intersection of the two straight segments from the linear fit occurs at $22.2 \mathrm{~Hz}$ and at a $\Delta R / \Delta F$ ratio $0.130 \mathrm{Ohm} \cdot \mathrm{Hz}^{-1}$.

To confirm that the observed difference in $\Delta R / \Delta F$ between high and low protein density on the surface could be attributed to the protein compact state, experiments using different protein concentrations and recording $\Delta F$ and $\Delta R$ simultaneously were performed (Fig. 5B). It is observed that at low protein concentrations the $\Delta R / \Delta F$ ratio increases as the protein concentration increases, associated to a higher viscoelasticity of the protein film, reaching a maximum of $0.124 \mathrm{Ohm} \cdot \mathrm{Hz}^{-1}$ at $0.075 \mu \mathrm{M}$ of protein. It is noteworthy that this $\Delta R / \Delta F$ value is comparable to that obtained from the intersection in Fig. 5A. This result shows that at low concentrations there are not enough neighbouring CTPR20-Cys molecules immobilized for them to assemble, as previously suggested. When the protein concentration is increased, a subsequent $\Delta R / \Delta F$ decrease is obtained until reaching a stable value of $0.06 \mathrm{Ohm} \cdot \mathrm{Hz}^{-1}$ at 
surface-saturating protein concentrations. The surface becomes more densely packed with CTPR20-Cys molecules, promoting lateral interactions and leading to an increase in the rigidity of the film. These results clearly suggest that the mode of attachment through the Cterminal Cys residue leads to a spatial arrangement, with parallel alignment between protein molecules, favouring the establishment of lateral protein interactions. In the case of CTPR20 protein without cysteine this analysis shows a different behaviour with a linear increase in the viscoelasticity of the deposit as the protein is adsorbed (Electronic supplementary material Figure S3).

The final step of this study is the functionalization of the protein monolayer with gold nanoparticles (GNPs) in order to monitor the templating capabilities of the CTPR monolayer. In addition, the attachment of GNPs will allow us to achieve a higher resolution characterization of the surface using SEM. GNPs stabilized with thioctic acid (TA-GNPs) displaying free carboxylic acid functions, required for the immobilization step, were used. As the CTPR20-Cys was selectively immobilized onto the gold surface through its single Cterminal cysteine, the $\mathrm{N}$-terminal moiety of the protein was selected as the anchoring point. The anchoring reaction is achieved through the formation of an amide bond between amine of the N-terminal of the CTPR20-Cys (previously immobilized on gold surface) and the carboxylic acid functions of TA-GNPs, which were activated in situ by EDC/NHS. After the reaction of the TA-GNPs with the gold surface coated with CTPR20-Cys, scanning electron microscope (SEM) was used to characterize the functional surfaces obtained. The AFM results showed the evidences of a monolayer of compacted CTPR-20-Cys (Fig. 4) onto gold surface and a homogenous coverage of protein over surfaces in the order of $\mu \mathrm{m}^{2}$. Each CTPR20-Cys molecule should be able to interact only with one GNP through an amide bond formation, and the area of the protein section is about $11 \mathrm{~nm}^{2}$, in the same range of the GNPs diameter. Therefore it is expected to observe a homogenous coverage of a single layer of GNPs over the CTPR20 surface.

Fig. 6A shows a significant GNPs surface coverage yield (clearer areas) in a highly packed distribution. The entire surface was not fully covered by GNPs but CTPR20-Cys can be observed on almost the whole surface (as black/grey background). An important observation is that when the functionalization with GNPs occurred, it formed preferentially a monolayer of GNPs with a high level of compaction, leading to islands of close-packed GNPs monolayers (Fig. 6B). At a higher magnification, the monolayer organization of the GNPs 
functionalized onto CTPR20 immobilized onto gold surface can be clearly confirmed (Fig. 6B). The controls of GNPs onto gold surface and GNPs onto CTPR20 without coupling agents gave very different results in SEM, with a majority of isolated GNPs aggregates and/or randomly dispersed (Electronic supplementary material). The comparison of both SEM (Fig. 6B) and AFM (Fig. 4B) at the same scale confirms the monolayer organization of the GNPs and that the same kind of organization is observed for the protein onto gold surface, presenting in both cases a high compaction. This was not observed when the GNPs were deposited on gold surfaces without prior CTPR20-Cys protein layer formation (Fig. S5 and S6), which corroborates that the CTPR20-Cys monolayer is templating the GNPs.

\section{Conclusions}

In conclusion, we have demonstrated that we can immobilize oriented long designed CTPR proteins on gold surface through thiol-gold specific interaction and that the protein modules present intrinsic lateral assembly properties. By controlled immobilization we generated a tightly packed protein SAM and characterized the packing by QCM, SPR, and AFM. We monitored the assembly transition by the change on the viscoelastic properties of the immobilized materials. It is clear that the oriented immobilization of the long protein arrays allows the arrangement of the modules and the formation of a rigid protein film on the surface. Regarding the mechanism of interaction between the proteins and the forces that drive the assembly, we can speculate based on the previously observed lateral packing of superhelices in solid films[35] and in crystal forms.[27] Long CTPR proteins are structurally rigid with anisotropic shape (Fig. 1), that allows for preferential alignment along one direction and lateral packing when the proteins are immobilized directionally oriented on a surface. The crystal packing provides hints that CTPR protein superhelices have a tendency to interact through salt bridges between positively and negatively charged residues on the protein surface.[58] Similar interactions can mediate side-to-side packing within monolayer. The functionalization with GNPs additionally confirms that most of the protein molecules are oriented as expected ( $\mathrm{N}$-terminal free) and in a monolayer disposition. This work is an example where the molecular understanding of the biomolecular blocks and the control of the immobilization and assembly process on surfaces can lead to the generation of materials with defined macroscopic properties. For example, by changing the protein packing density, the rigidity of the protein film can be tuned at will. Additionally, these ordered and structured biocompatible surfaces can be functionalized by introducing unique groups with atomic 
resolution at defined positions in the protein. The results of this work show the potential of these designed scaffolds as platforms for patterning GNPs. The assembly of GNPs into closely packed thin films has great interest because of their potential applications in electronics, nanophotonics, data storage, and plasmonics.[59] The templating strategy described here is versatile and can be easily applied to a variety of nanoparticles and other molecules for the generation of closely packed arrays. The application of these biomolecular templates can result in novel types of hybrid materials within the bottom-up approaches.

\section{Materials and methods}

\subsection{Protein design and purification}

CTPR20 protein with a single cysteine at the C-terminal was expressed as His-tagged fusion and purified using standard affinity chromatography methods as previously described. $[13,27,44]$ The protein was dialyzed into PBS buffer $(150 \mathrm{mM} \mathrm{NaCl}, 50 \mathrm{mM}$ phosphate buffer $\mathrm{pH}$ 7.4) and stored frozen at $-20^{\circ} \mathrm{C}$. The protein concentration was determined by $U V$ absorption using a Cary $50 U V$-visible spectrophotometer (Varian) at $280 \mathrm{~nm}$ using the extinction coefficient calculated from the amino acid composition.[60]

\subsection{Protein reduction for gold surface immobilization experiments}

Prior to any immobilization experiment on gold, purified CTPR20-Cys at a protein concentration of about $23 \mu \mathrm{M}$ was freshly reduced with $1 \mathrm{mM}$ dithiothreitol (DTT) during 20 minutes to ensure full reduction of the sample. DTT was removed by buffer exchange over a NAP-5 column (GE Healthcare Life Science) in PBS-2 buffer with lower salt and buffer concentration $(10 \mathrm{mM} \mathrm{NaCl}, 10 \mathrm{mM}$ phosphate buffer $\mathrm{pH}$ 7.4) or water. Protein fractions without DTT are collected at $4^{\circ} \mathrm{C}$ and their concentration measured by $U V$ absorption spectroscopy.

\subsection{Quartz Crystal Microbalance (QCM)}

QCM is an extremely sensitive mass sensor capable of providing real-time monitoring of immobilization events in the nanogram range and determination of kinetic rate constant. QCM measures frequency changes in the resonant frequency of the quartz crystal according 
to Sauerbrey's equation (Eq 1), where a decrease in mass corresponds to an increase in frequency and vice versa. Moreover, QCM with motional resistance monitoring also allows obtaining information related to the structure, conformation, and interactions of the molecules on the surface through the acoustic dissipation. In situ mass changes were measured with a SRS QCM200 Quartz Crystal Microbalance from SRS Instruments (Sunnyvale, CA., USA) with AT-cut quartz crystals ( $5 \mathrm{MHz}$ ) of $25 \mathrm{~mm}$ diameter with gold electrodes deposited over a Chromium adhesion layer. An asymmetric electrode format was used with the side having the larger gold area facing the solution. The electroactive working area (front side) was $1.370 \mathrm{~cm}^{2}$ and the piezoelectric area (backside) was $0.317 \mathrm{~cm}^{2}$.

Prior to use, the quartz crystals were cleaned by exposure to "piranha" solution $(3: 1$ concentrated $\mathrm{H}_{2} \mathrm{SO}_{4} / 30 \% \mathrm{H}_{2} \mathrm{O}_{2}$ ) followed by exhaustive rinsing with distilled water and a final rinse with ethanol/water (2:1). Caution: piranha solution reacts violently with most organic materials and must be handled with extreme care. The quartz crystal resonator was set in a probe made of Teflon and immersed in water-jacketed beaker thermostated at $25^{\circ} \mathrm{C}$. The crystals sensitivity was $17.6810^{-9} \mathrm{~g} \cdot \mathrm{Hz}^{-1} \cdot \mathrm{cm}^{-2} \cdot[61-63]$

Under constant flow protein samples in PBS-2 buffer at concentrations from $5 \mathrm{nM}$ to $2.5 \mu \mathrm{M}$ are injected in the system and both the changes in motional frequency and in resistance were recorded until saturation is reached.

Sauerbrey's equation describes the correlation between frequency changes in the resonant frequency of the quartz crystal and the immobilize mass for QCM experiments:

$$
\Delta m=-C_{\mathrm{f}} F
$$

\section{Equation 1}

where $\Delta m$ is the mass change $\left(\mathrm{ng} \cdot \mathrm{cm}^{-2}\right), C_{\mathrm{f}}\left(17.7 \mathrm{ng} \mathrm{Hz} \mathrm{Hz}^{-1} \cdot \mathrm{cm}^{-2}\right)$ the proportionality constant for the 5.0 MHz crystals used in this study, and $\Delta F$ the frequency change (in hertz). Although this equation rigorously applies to the system in air and mass additions forming an evenly distributed rigid layer on the active sensor area,[64] it is widely accepted to estimate the adsorbed mass of protein in liquid environments.[65-67] Sauerbrey equation can therefore be reliably used to measure the mass of thin, evenly distributed, rigid and non-porous overlayers even in the liquid phase.[64] In this way, it has been reported that the linearity between adsorbed mass and frequency response may persist under certain conditions.[68]

Assuming that the immobilization process is kinetically controlled the frequency curves were fit to a first-order kinetics equation: 


$$
\Delta F=-\Delta F_{\max }\left(1-\mathrm{e}^{-k t}\right)
$$

Equation 2

where $\Delta F$ is the frequency change (in hertz), $\Delta F_{\max }$ the frequency change between the initial and the steady-state frequencies, and $k$ the first-order rate constant $\left(\mathrm{min}^{-1}\right)$.

The frequency-time data for CTPR20 can be well fitted to a first order doubleexponential decay with two adsorption processes, one fast decay rate and a subsequent slower adsorption:

$$
\Delta F=\Delta F_{\text {fast }} \exp \left(-k_{\text {fast }} t\right)+\Delta F_{\text {slow }} \exp \left(-k_{\text {slow }} t\right)+\Delta F_{\max }
$$

Equation 3

From the $\Delta F_{\max }$ at the equilibrium and using Sauerbrey's equation (Equation 1) the total mass of protein can be estimated assuming that the frequency decrease is only due to the change in mass arising from the adsorption of the protein immobilized on the gold electrode surface.

From the frequency change at the equilibrium $\left(\Delta \mathrm{F}_{\max }\right)$, the total amount of protein immobilized per surface area can be calculated at the different protein injections. The equilibrium protein coverage values were represented versus protein concentrations and fitted to a Langmuir adsorption isotherm:

\section{Equation 4}

$$
\Gamma_{e}=\frac{\Gamma_{s} K_{a} \mathrm{C}_{s}}{1+K_{a} \mathrm{C}_{s}}
$$

where $\mathrm{K}_{\mathrm{a}}$ is the thermodynamic binding constant, $\mathrm{C}_{\mathrm{s}}$ is the bulk protein concentration, and $\Gamma_{\mathrm{e}}$ and $\Gamma_{\mathrm{s}}$ are the equilibrium and saturation protein coverage.

\subsection{Surface plasmon resonance (SPR) measurements}

A gold film of approximately $30 \mathrm{~nm}$ was first deposited on sodalime glass substrate (1 $\mathrm{mm}$ thick) by electron beam evaporation in a Molecular Beam Epitaxy system with a base pressure of $\mathrm{Pb}=1 \times 10-10$ Torr. The $\mathrm{SPR}$ spectrum of the gold surface was measured to calculate the exact thickness of the film. Then, a CTPR20-Cys protein solution at $2.5 \mu \mathrm{M}$ concentration was deposited on the gold surface. After incubation for 30 minutes at room temperature the SPR spectrum of the protein film in solution was acquired. 
SPR spectra were acquired using the Kretschmann-Raether configuration, [69, 70] with a home-made device described elsewhere.[50] SPR was excited with a $632.8 \mathrm{~nm}$ laser. The angular response of the photodiode was corrected as described before.[51] For each sample 4 scans were recorded from different spatial locations. The spectra shown here correspond to the average (with the line thickness being the standard deviation). Any possible drift in the motor position was corrected by fixing the position of the critical angle to $39.5 \mathrm{deg}$; this value depends only on the glass and the surrounded air, and it is independent of the gold and protein films. To calculate the total mass of protein immobilized on the gold surface, a shift of 0.1 deg on the resonance angle is related to a mass of $1 \mathrm{ng} \cdot \mathrm{mm}^{-2}$, as described before for proteins.[71] SPR curve simulation was carried out using Winspall freeware by RES-TEC (http://www.res-tec.de/applications.html) including the correction of refraction for triangular prisms.

\subsection{Atomic force microscopy (AFM)}

For AFM measurements CTPR20-Cys protein was immobilized on gold surfaces. The gold substrates were commercial gold-coated borosilicate glass substrates (Gold Arrandee ${ }^{\mathrm{TM}}$ ) annealed using a Bunsen burner prior to use. First, we monitored gold surfaces under different annealing conditions without protein to achieve sub-nanometric roughness and ensure that the surface roughness is small enough to clearly image the nanometric-scale protein molecules. CTPR20-Cys freshly reduced protein as described above was incubated on the gold surface during 1 hour. After incubation the surface is thoroughly washed with water.

The protein coated surface is imaged in solution using a Nanotec Cervantes system in jumping mode.[72] Olympus standard silicon nitride probes of $0.05 \mathrm{~N} \cdot \mathrm{m}^{-1}$ and $18 \mathrm{kHz}$ (OMCL-RC800PSA) were employed. Images were processed using the WSxM software (Nanotech Electronica, Madrid, Spain http://www.nanotec.es/).[73]

\subsection{Profilometry measurements}

CTPR20-Cys protein was immobilized on gold surfaces using the same protocol used for the generation of the AFM samples. Three different layers were obtained by incubation of $2.5 \mu \mathrm{M}$ protein. The layer thickness was measured using a "Dektak XT" mechanical profilometer with a $2.5 \mu \mathrm{m}$ radio stylus and $1 \mathrm{mg}$ force (the minimal force). 


\subsection{Gold nanoparticle (GNP) synthesis}

Gold nanoparticles were synthesized following the well-established Turkevich protocol[74] with slight adjustments as described previously.[75] Briefly, $100 \mathrm{~mL}$ of an aqueous solution of $\mathrm{HAuCl}_{4}$ (Sigma-Aldrich) at $1 \mathrm{mM}$ was refluxed at $140^{\circ} \mathrm{C}$ and reduced for 15 min with $10 \mathrm{~mL}$ of an aqueous solution of sodium citrate $(55 \mathrm{mM})$. The reaction led rapidly to a red-wine solution of citrate based GNPs. The sample is then cooled to room temperature and filter through $0.2 \mu \mathrm{m}$ pore-size filter to eliminate large aggregates. The final concentration of GNPs (9 $\mathrm{nM})$ was determined by $U V / V I S$ spectrometry using an extinction coefficient at $520 \mathrm{~nm}, \varepsilon=2.7 \times 10^{8} \mathrm{M}^{-1} \cdot \mathrm{cm}^{-1}$. The as-modified Turkevich protocol for the synthesis of GNPs leads to a well-dispersed colloidal suspension of citrate-stabilized GNPs with an average diameter of $12.4 \pm 1.2 \mathrm{~nm}$ (measured by TEM, Fig. S6). A second step of ligand exchange with thioctic acid was carried out to introduce a more stable surfactant with carboxylic groups. To $10 \mathrm{~mL}$ of GNPs at $9 \mathrm{nM}$ was added $1.15 \mathrm{~mL}$ of thioctic acid at $10 \mathrm{mM}$ in ethanol to react overnight at room temperature. Then, sample was centrifuged $40 \mathrm{~min}$ at $10000 \mathrm{xg}$ and supernatant discarded. The red-wine pellet was redispersed in $7 \mathrm{~mL}$ milliQ water, resulting in a light purple suspension. Then, drops of $\mathrm{NaOH} 10 \mathrm{mM}$ were added until the solution turned to red-wine color, indicating a good colloidal dispersion of thioctic acid stabilized GNPs (TA-GNPs) due to negative charges of the carboxylic acids.

\subsection{GNPs conjugation with CTPR20-Cys immobilized onto gold surface}

The conjugation between TA-GNPs and CTPR20-Cys, previously immobilized onto gold surface by the C-terminal Cys residue, was carried out through the reaction between carboxylic acid functions of the GNPs ligands and the free exposed terminal amine of the protein. Frist, $9 \mu \mathrm{L}$ of ethyl(dimethylaminopropyl) carbodiimide (EDC, $1 \mathrm{mM}$ ) and $6 \mu \mathrm{L}$ of N-hydroxysuccinimide (NHS, $1 \mathrm{mM}$ ) were added to $1 \mathrm{~mL}$ of TA-GNPs at $30 \mathrm{nM}$ for $10 \mathrm{~min}$ to perform the activation of the carboxylic groups. Then, $200 \mu \mathrm{L}$ of activated TA-GNPs were incubated onto the gold surface covered by CTPR20-Cys during 3 days at room temperature. Controls of the same experiment without adding EDC/NHS coupling agents and the immobilization of TA-GNPs onto protein-free gold surface were also carried out under the same experimental conditions.

After the incubation time, the liquid phase was removed from the gold surface and the surface was washed several times with MilliQ water and dried under nitrogen flux. 


\subsection{Scanning Electron Microscopy (SEM)}

SEM measurements of the surfaces with gold nanoparticles were carried out at low acceleration voltages with an ultra high resolution Scanning Electron Microscope, Auriga Cross Beam FIB-SEM from Carl Zeiss equipped with a Gemini electron beam column and inlens detector technology, at IMDEA Nanociencia. The samples were deposited on gold substrates and imaged using a 2-3 kV beam acceleration voltages. 


\section{Acknowledgements}

Financial support for this research was obtained from the Spanish Ministerio de Economía y Competitividad (BIO2012-34835 A.L.C. and J.M.A.), the European Commission International Reintegration Grant (IRG-246688) (A.L.C.) and Marie Curie COFUND “AMAROUT-Europe" Programme (A.L.C.). S.H.M. thanks IMDEA-Nanociencia for financial support through an "Ayuda de Iniciación a la Investigación" fellowship and the Basque Government for financial support (PhD Scholarship). JMA acknowledges research funding by a "Ramon y Cajal" contract from the Spanish Ministry of Science and Innovation. We thank Prof. Encarnación Lorenzo for providing QCM measurement facilities.

\section{References}

[1] A.P. Alivisatos, Nanocrystals: Building blocks for modern materials design, Endevour, 21 (1997) 56-60.

[2] T. Pellegrino, S. Kudera, T. Liedl, A. Muñoz Javier, L. Manna, W.J. Parak, On the Development of Colloidal Nanoparticles towards Multifunctional Structures and their Possible Use for Biological Applications., Small, 1 (2005) 48-63.

[3] V.F. Puntes, K.M. Krishnan, A.P. Alivisatos, Colloidal Nanocrystal Shape and Size Control: The Case of Cobalt, Science, 291 (2001) 2115-2117.

[4] A.M. Jackson, J.W. Myerson, F. Stellacci, Spontaneous assembly of subnanometreordered domains in the ligand shell of monolayer-protected nanoparticles., Nat. Materials, 3 (2004) 330-336.

[5] W.J. Parak, D. Gerion, T. Pellegrino, D. Zanchet, C. Micheel, S.C. Williams, R. Boudreau, M.A. Le Gros, C.A. Larabell, A.P. Alivisatos, Biological applications of colloidal nanocrystals, Nanotechnology, 14 (2003) R15.

[6] G.A. Devries, M. Brunnbauer, Y. Hu, A.M. Jackson, B. Long, B.T. Neltner, O. Uzun, B.H. Wunsch, F. Stellacci, Divalent metal nanoparticles, Science, 315 (2007) 358-361.

[7] E. Gazit, Bioinspired chemistry: Diversity for self-assembly, Nat Chem, 2 (2010) 10101011.

[8] D.N. Woolfson, Z.N. Mahmoud, More than just bare scaffolds: towards multi-component and decorated fibrous biomaterials, Chem Soc Rev, 39 (2010) 3464-3479.

[9] R.V. Ulijn, D.N. Woolfson, Peptide and protein based materials in 2010: from design and structure to function and application, Chem Soc Rev, 39 (2010) 3349-3350.

[10] K. Rajagopal, J.P. Schneider, Self-assembling peptides and proteins for nanotechnological applications, Curr Opin Struct Biol, 14 (2004) 480-486.

[11] S.A. Maskarinec, D.A. Tirrell, Protein engineering approaches to biomaterials design., Curr. Opin. Biotechnol., 16 (2005) 422-426.

[12] Langer R, T. DA., Designing materials for biology and medicine., Nature, 428 (2004) 487-492.

[13] T. Kajander, A.L. Cortajarena, L. Regan, Consensus design as a tool for engineering repeat proteins, Methods Mol. Biol., 340 (2006) 151-170.

[14] D.M. Ridgley, B.G. Freedman, P.W. Lee, J.R. Barone, Genetically encoded selfassembly of large amyloid fibers, Biomater. Sci., 2 (2014) 560-566. 
[15] M. Sarikaya, C. Tamerler, D. Schwartz, F. NBaneyx, Materials Assembly and Formation using Engineered Polypeptides., Annu. Rev. Mater. Res., 34 (2004) 373-408.

[16] P.F. McMillan, New materials from high-pressure experiments, Nature Materials, 1 (2002) 19 - 25.

[17] M. Zin, H. Ma, M. Sarikaya, J. AK, Assembly of gold nanoparticles using genetically engineered polypeptides., Small, 1 (2005) 698-702.

[18] V.V. Zhirnov, D.J.C. Herr, New frontiers: Self-assembly in nanoelectronics., IEEE Computer, 34 (2001) 33-43.

[19] Asbahi M., Lim KT, Wang F, Duan H, Thiyagarajah N, Ng V, Y. JK., Directed selfassembly of densely packed gold nanoparticles., Langmuir, 28 (2012) 16782-16787.

[20] C. Tamerler, M. Sarikaya, Molecular biomimetics: nanotechnology and bionanotechnology using genetically engineered peptides, Philos Trans A Math Phys Eng Sci., 367 (2009) 1705-1726.

[21] L. Hu, Chen M, Fang X, W. L., Oil-water interfacial self-assembly: a novel strategy for nanofilm and nanodevice fabrication, Chem Soc Rev, 41 (2012) 1350-1362.

[22] D. Romera, P. Couleaud, S.H. Mejias, A. Aires, A.L. Cortajarena, Biomolecular templating of functional hybrid nanostructures using repeat protein scaffolds, Biochem. Soc. Trans., 34 (2015) 825-831.

[23] L. D'Andrea, L. Regan, TPR proteins: the versatile helix, Trends Biochem. Sci., 28 (2003) 655-662.

[24] E. Main, A. Lowe, S. Mochrie, S. Jackson, L. and Regan, A recurring theme in protein engineering: the design, stability and folding of repeat proteins., Curr. Opin. Struc. Biol., 15 (2005) 464-471.

[25] T.Z. Grove, L. Regan, New materials from proteins and peptides, Curr. Opin. Struct. Biol., 22 (2012) 451-456.

[26] E.R. Main, J.J. Phillips, C. Millership, Repeat protein engineering: creating functional nanostructures/biomaterials from modular building blocks, Biochem. Soc. Trans., 41 (2013) $1152-1158$.

[27] T. Kajander, A.L. Cortajarena, S.G. Mochrie, L. Regan, Structure and stability of a consensus TPR superhelix, Acta Crystallogr. D, D63 (2007) 800-811.

[28] T. Kajander, A.L. Cortajarena, E.R. Main, S.G. Mochrie, L. Regan, A new folding paradigm for repeat proteins., J. Am. Chem. Soc., 127 (2005) 10188-10190.

[29] T.Z. Grove, M. Hands, L. Regan, Creating novel proteins by combining design and selection, Protein Eng Des Sel, 23 (2010) 449-455.

[30] A.L. Cortajarena, L. Regan, Ligand binding by TPR domains, Protein Sci., 15 (2006) 1193-1198.

[31] A.L. Cortajarena, S.G. Mochrie, L. Regan, Modulating repeat protein stability: The effect of individual helix stability on the collective behavior of the ensemble, Protein Sci., 20 (2011) 1042-1047.

[32] T.Z. Grove, C.O. Osuji, J.D. Forster, E.R. Dufresne, L. Regan, Stimuli-responsive smart gels realized via modular protein design, J Am Chem Soc, 132 (2010) 14024-14026.

[33] A. Sanz de Leon, J. Rodriguez-Hernandez, A.L. Cortajarena, Honeycomb patterned surfaces functionalized with polypeptide sequences for recognition and selective bacterial adhesion, Biomaterials, 34 (2013) 1453-1460.

[34] M. Palacios-Cuesta, A.L. Cortajarena, O. García, J. Rodríguez- Hernández, Versatile functional microstructured polystyrene-based platforms for protein patterning and recognition, Biomacromolecules, 14 (2013) 3147-3154.

[35] T.Z. Grove, L. Regan, A.L. Cortajarena, Nanostructured functional films from engineered repeat proteins, J. R. Soc. Interface., 10 (2013) 20130051. 
[36] J.J. Phillips, C. Millership, E.R. Main, Fibrous nanostructures from the self-assembly of designed repeat protein modules., Angew Chem, 51 (2012) 13132-13135.

[37] S.H. Mejias, B. Sot, R. Guantes, A.L. Cortajarena, Controlled nanometric fibers of selfassembled designed protein scaffolds, Nanoscale, In press (2014).

[38] A.L. Cortajarena, J. Wang, L. Regan, Crystal structure of a designed TPR module in complex with its peptide-ligand, FEBS J., 277 (2010) 1058-1066.

[39] N.A. Carter, T.Z. Grove, Repeat-proteins films exhibit hierarchical anisotropic mechanical properties., Biomacromolecules, 16 (2015) 706-714.

[40] A.L. Cortajarena, L. Regan, Calorimetric study of a series of designed repeat proteins: modular structure and modular folding., Protein Sci., 20 (2011) 341-352.

[41] Y.-S. Shon, H.Y. Choi, M.S. Guerrero, C. Kwon, Preparation of Nanostructured Film Arrays for Transmission Localized Surface Plasmon Sensing., Plasmonics, 4 (2009) 95-105

[42] Chen MC, Yang YL, Chen SW, Li JH, Aklilu M, T. Y., Self-assembled monolayer immobilized gold nanoparticles for plasmonic effects in small molecule organic photovoltaic., ACS Appl Mater Interfaces, 5 (2013) 511-517.

[43] Zhang X, Zhang J, Wang H, Hao Y, Zhang X, Wang T, Wang Y, Zhao R, Zhang H, Y. B., Thermal-induced surface plasmon band shift of gold nanoparticle monolayer: morphology and refractive index sensitivity., Nanotechnology, 21 (2010) 465702.

[44] A.L. Cortajarena, G. Lois, E. Sherman, C.S. O'Hern, L. Regan, G. Haran, Extensive nonnative polyproline II secondary structure induces compaction of a protein's denatured state., J. Mol. Biol., 382 (2008) 203-212.

[45] J. Love, Estroff LA, Kriebel JK, Nuzzo RG, W. GM., Self-assembled monolayers of thiolates on metals as a form of nanotechnology., Chem. Rev., 105 (2005) 1103-1169.

[46] J.L. Wilbur, G.M. Whitesides, Self-Assembly and Self-Assembled Monolayers in Microand Nanofabrication, in: G. Timp (Ed.) Nanotechnology, Springer1999, pp. 331-369.

[47] L.H. Dubois, R.G. Nuzzo, Synthesis, structure and properties of model organic surfaces., Annu. Rev. Phys. Chem., 43 (1992) 437-463.

[48] S. Nakata, N. Kido, M. Hayashi, M. Hara, H. Sasabe, T. Sugawara, T. Matsuda, Chemisorption of proteins and their thiol derivatives onto gold surfaces: characterization based on electrochemical nonlinearity, Biophysical Chemistry, 62 (1996) 63-72.

[49] V. Silin, H. Weetall, D.J. Vanderah, SPR Studies of the Nonspecific Adsorption Kinetics of Human IgG and BSA on Gold Surfaces Modified by Self-Assembled Monolayers (SAMs). J. Colloid and Interface Science, 185 (1997) 94-103.

[50] A. Serrano, O. Rodríguez de la Fuente, M.A. García, Extended and localized surface plasmons in annealed Au films on glass substrates, J. Appl. Phys., 108 (2010) 074303.

[51] F. Galvez, C. Monton, A. Serrano, I. Valmianski, J. de la Venta, I.K. Schuller, M.A. Garcia, Effect of photodiode angular response on surface plasmon resonance measurements in the Kretschmann-Raether configuration., Rev Sci Instrum., 83 (2012) 093102.

[52] K.M.M. Aung, X. Ho, X. Su, DNA assembly on streptavidin modified surface: A study using quartz crystal microbalance with dissipation or resistance measurements, Sensors and Actuators B: Chemical, 131 (2008) 371-378.

[53] Bustamante C, Vesenka J, Tang CL, Rees W, Guthold M, K. R., Circular DNA molecules imaged in air by scanning force microscopy., Biochemistry, 31 (1992) 22-26.

[54] G. Lee, K. Abdi, Y. Jiang, P. Michaely, V. Bennett, P.E. Marszalek, Nanospring behaviour of ankyrin repeats., Nature, 440 (2006) 246-249.

[55] Cohen SS, Riven I, Cortajarena AL, De Rosa L, D'Andrea LD, Regan L, H. G., Probing the Molecular Origin of Native-State Flexibility in Repeat Proteins., J. Am. Chem. Soc., 137 (2015) 10367-10373. 
[56] T. Matsuura, H. Tanaka, T. Matsumoto, T. Kawai, Atomic force microscopic observation of Escherichia coli ribosomes in solution, Bioscience, biotechnology, and biochemistry, 70 (2006) 300-302.

[57] R. Lucklum, C. Behling, P. Hauptmann, Role of mass accumulation and viscoelastic film properties for the response of acoustic-wave-based chemical sensors, Anal. Chem., 71 (1999) 2488-2496.

[58] T. Kajander, A.L. Cortajarena, S.G. Mochrie, L. Regan, Structure and stability of a consensus TPR superhelix, Acta Crystallographica, D63 (2007) 800-811.

[59] D. Talapin, J.-S. Lee, M. Kovalenko, E. Shevchenko, Prospects of Colloidal Nanocrystals for Electronic and Optoelectronic Applications, Chemical reviews, 110 (2010) 389-458.

[60] C.N. Pace, F. Vajdos, L. Fee, G. Grimsley, T. Gray, How to measure and predict the molar absorption coefficient of a protein, Protein Sci., 4 (1995) 2411-2423.

[61] J.M. Abad, M. Velez, C. Santamaria, J.M. Guisan, P.R. Matheus, L. Vazquez, I. Gazaryan, L. Gorton, T. Gibson, V.M. Fernandez, Immobilization of peroxidase glycoprotein on gold electrodes modified with mixed epoxy-boronic Acid monolayers, J Am Chem Soc, 124 (2002) 12845-12853.

[62] M. Darder, E. Casero, D.J. Díaz, H.D. Abruña, F. Pariente, E. Lorenzo, Concentration Dependence of Aggregate Formation upon Adsorption of 5-(Octyldithio)-2-nitrobenzoic Acid on Gold Electrodes, Langmuir, 16 (2001) 9804-9811.

[63] J. Madoz-Gurpide, J.M. Abad, J. Fernandez-Recio, M. Velez, L. Vazquez, C. GomezMoreno, V.M. Fernandez, Modulation of Electroenzymatic NADPH Oxidation through Oriented Immobilization of Ferredoxin:NADP+ Reductase onto Modified Gold Electrodes, J. Am. Chem. Soc., 122 (2000) 9808-9817.

[64] M. Rodahl, F. Höök, C. Fredriksson, C.A. Keller, A. Krozer, P. Brzezinski, M. Voinova, B. Kasemo, Simultaneous frequency and dissipation factor QCM measurements of biomolecular adsorption and cell adhesion., Faraday Discuss., 107 (1997) 229-246.

[65] S. Bruckenstein, M. Shay, Experimental aspects of use of the quartz crystal microbalance in solution, Electrochimica Acta, 30 (1985) 1295-1300.

[66] M.D. Ward, D.A. Buttry, In situ interfacial mass detection with piezoelectric transducers., Science, 249 (1990) 1000-1007.

[67] M. Rodahl, B. Kasemo, On the measurement of thin liquid overlayers with the quartz crystal microbalance, Sens. Actuators A, 54 (1996) 448-462.

[68] J. Rickert, A. Brecht, W. Göpel, QCM Operation in Liquids: Constant Sensitivity during Formation of Extended Protein Multilayers by Affinity., Anal. Chem., 69 (1997) 1441-1448.

[69] W. Knoll, Interfaces and thin films as seen by bound electromagnetic waves. , Annu Rev Phys Chem. , 49 (1998) 569-638.

[70] H. Raether, Surface Plasmons on Smooth and Rouge Surfaces and on Gratings, Berlin, 1998.

[71] E. Stenberg, B. Persson, H. Roos, C. Urbaniczky, Quantitative determination of surface concentration of protein with surface plasmon resonance using radiolabeled proteins, Journal of Colloid and Interface Science, 143 (1991) 513-526.

[72] P.J. de Pablo, J. Colchero, J. Gomez-Herrero, A.M. Baro, Jumping mode scanning force microscopy, Appl. Phys. Lett. , 73 (1998) 3300-3302.

[73] Horcas I., Fernández R., Gómez-Rodríguez J. M., Colchero J., Gómez-Herrero J., A.M. Baro, WSXM: A software for scanning probe microscopy and a tool for nanotechnology., Rev. Sci. Instrum. , 78 (2007) 13705-13713.

[74] J. Turkevich, P.C. Stevenson, J. Hillier, A study of the nucleation and growth processes in the synthesis of colloidal gold, Discuss. Faraday Soc., 11 (1951) 55-75. 
[75] A. Latorre, C. Posch, Y. Garcimart, M. Sanlorenzo, I. Vujic, M. Zekhtser, K. Rappersberger, S. Ortiz-urda, DNA and aptamer stabilized gold nanoparticles for targeted delivery of anticancer therapeutics, Nanoscale, 6 (2014) 7436-7442.

\section{Figures}
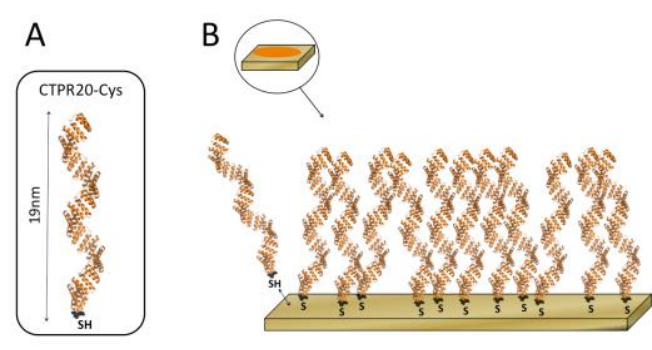

C
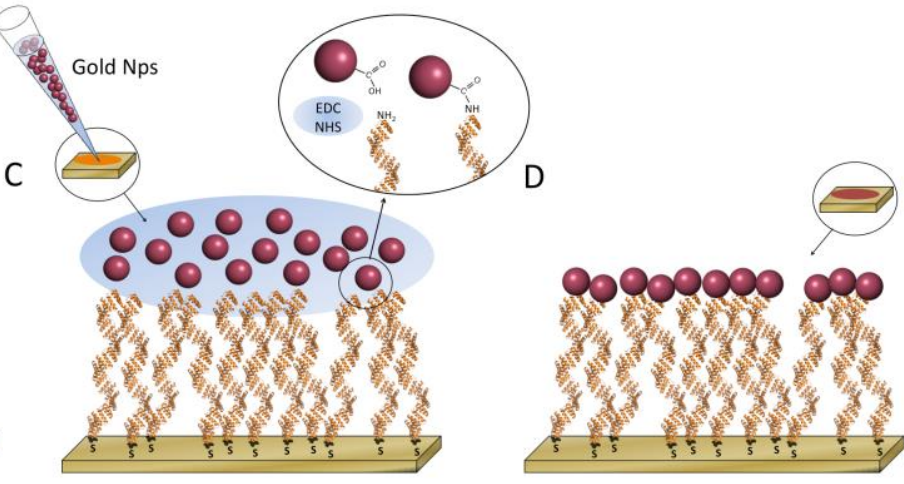

Fig. 1. A. Representation of the crystal structure of CTPR20-Cys protein. B. Schematic representation of CTPR20-Cys adsorption onto gold surface through thiol-gold interactions. C. Overview of the functionalization process of gold-adsorbed CTPR20-Cys with gold nanoparticles (GNPs) through amide bond formation in the presence of EDC and NHS. D. Schematic representation of the GNPs monolayer patterned by the previously adsorbed monolayer of CTPR20-Cys onto gold surface. 

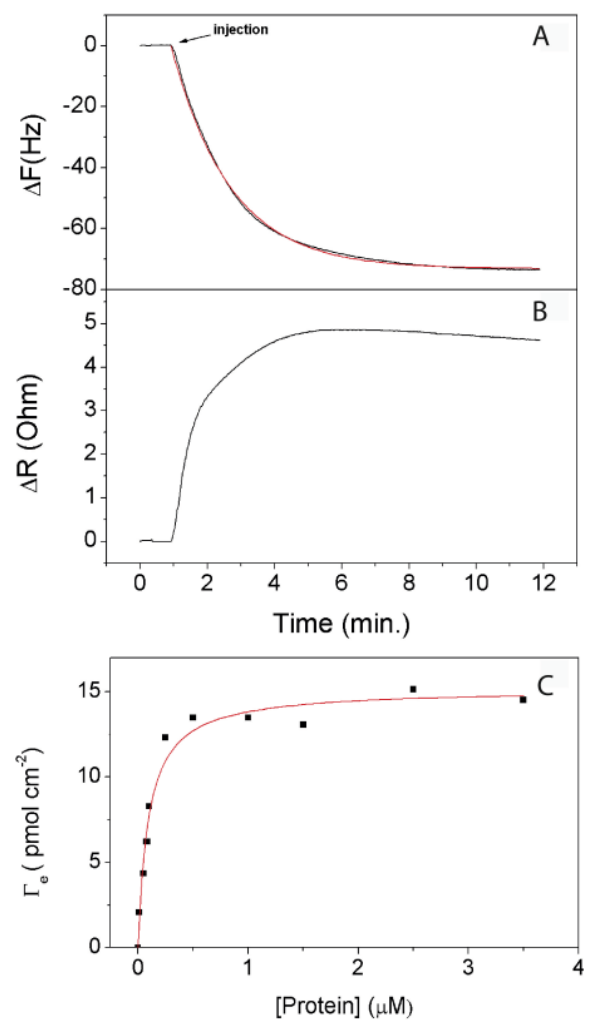

Fig. 2. CTPR20-Cys adsorption on gold surface monitored by quartz crystal microbalance. A. Adsorption kinetics of CTPR20-Cys onto quartz crystals with gold electrodes at $2.5 \square \mathrm{M}$ protein concentration. The frequency changes of the quartz crystal resonator are recorded upon protein injection. The curve was fit to a first-order kinetics equation (solid line), obtaining $-73.2 \mathrm{~Hz}$ and 0.57 $\min ^{-1}$ values for $-\Delta F_{\max }$ and $k$, respectively. B. Simultaneous resistance increase recorded by QCM upon CTPR20-Cys protein injection. C. Equilibrium surface coverage by QCM. Equilibrium surface coverage of CTPR20-Cys adsorbed on gold surface represented versus the protein concentration and fitted using a Langmuir adsorption isotherm. The calculated value for $\Gamma \mathrm{s}$ was $15.2 \times 10^{-12} \mathrm{~mol} \cdot \mathrm{cm}^{-2}$ for CTPR20-Cys. 

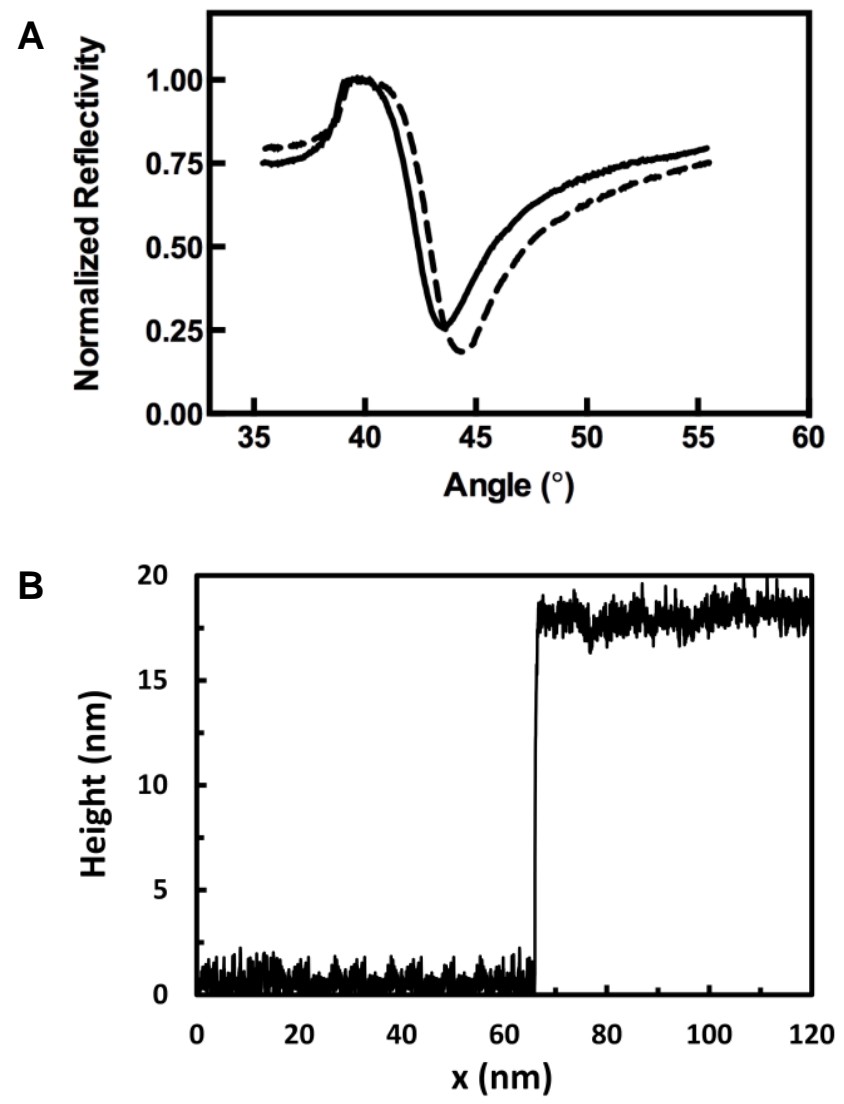

Fig. 3. A. Surface protein coverage by surface plasmon resonance. SPR spectra of bared gold surface (solid line) and CTPR20-Cys deposited on the gold surface (dashed line). The protein spectrum fits with a gold film thickness of $32 \pm 2 \mathrm{~nm}$, a protein coverage of approximately $1000 \mathrm{ng} \cdot \mathrm{cm}^{-2}$. B. Height profile of the CTPR20-Cys monolayer deposited on the gold surface acquired by a stylus profilometer. The thickness of the protein layer was $17.93 \pm 1.05 \mathrm{~nm}$. 

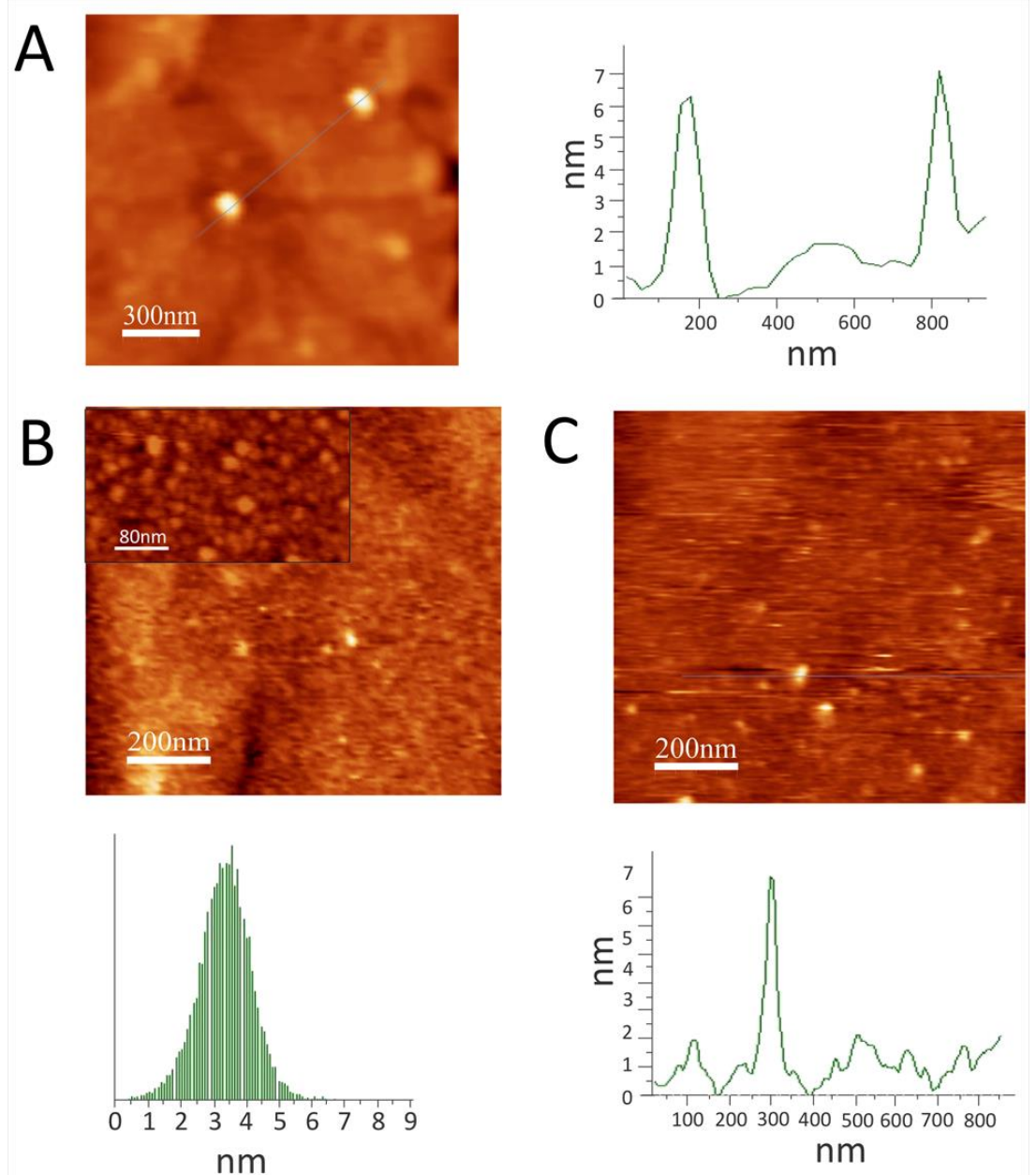

Fig. 4. AFM image of CTPR20-Cys protein immobilized on gold surfaces. A. AFM image of individual CTPR20-Cys molecules deposited on gold surface at $10 \mathrm{nM}$ protein concentration after 1 hour incubation at room temperature. Each bright spot corresponds to a single protein. The right panel shows the height profile of two single protein molecules. Note that the nanometer-scale widths obtained by AFM are not reliable due to the size and shape of the contacting probe. B. AFM image of a large area of completely saturated CTPR20-Cys monolayer deposited on gold surface at $2.5 \mu \mathrm{M}$ protein concentration. The inset shows a zoom-in image in which individual protein units can be observed. The panel below shows the global roughness analysis of the image that showed an RMS roughness of $0.86 \mathrm{~nm}$ and an average size of $3.42 \mathrm{~nm}$. C. AFM image of the same region as image B after the addition of DTT to the sample in order to remove the CTPR20-Cys. The panel below shows the height profile along the line to show a single protein molecule. 

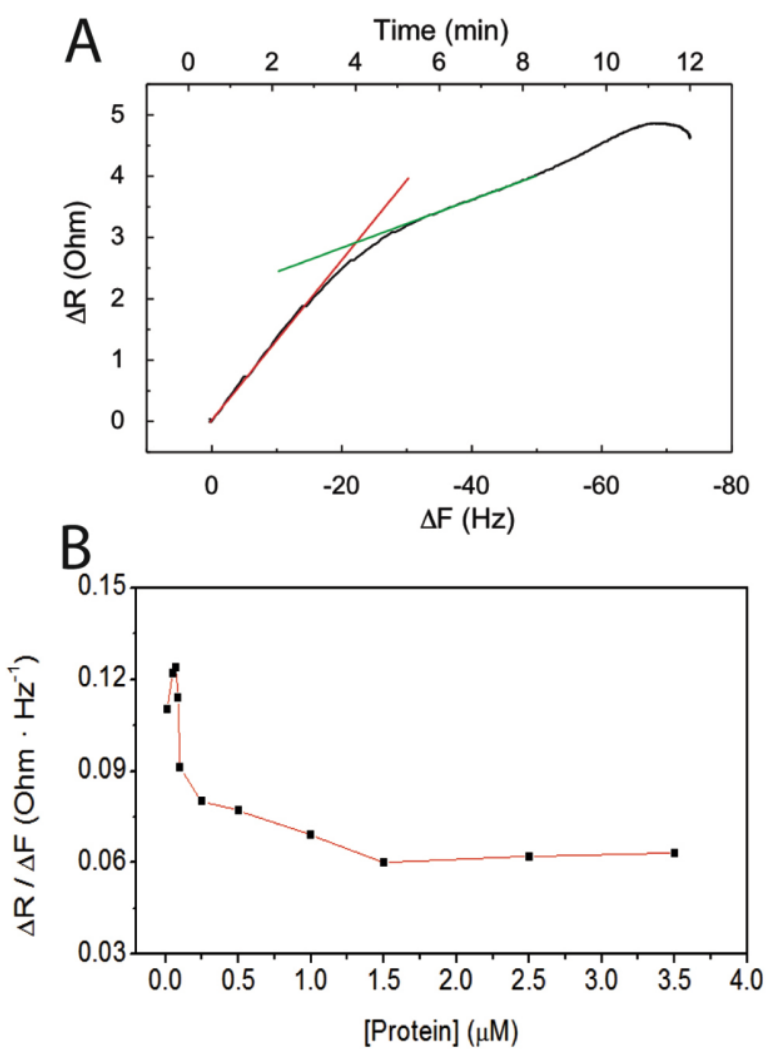

Fig. 5. Viscoelastic properties of deposited protein material measured by QCM. A. The change in resistance during the course of protein immobilization and assembly kinetics $v s$. the change in frequency for CTPR20-Cys at $2.5 \mu \mathrm{M}$ protein concentration. For CTPR20-Cys two regions are observed. The fit to linear equations with two different slopes are shown. B. The change in resistance divided by the change in frequency $(\Delta \mathrm{R} / \Delta \mathrm{F})$ for different protein concentrations of CTPR20-Cys measured after steady state was reached.
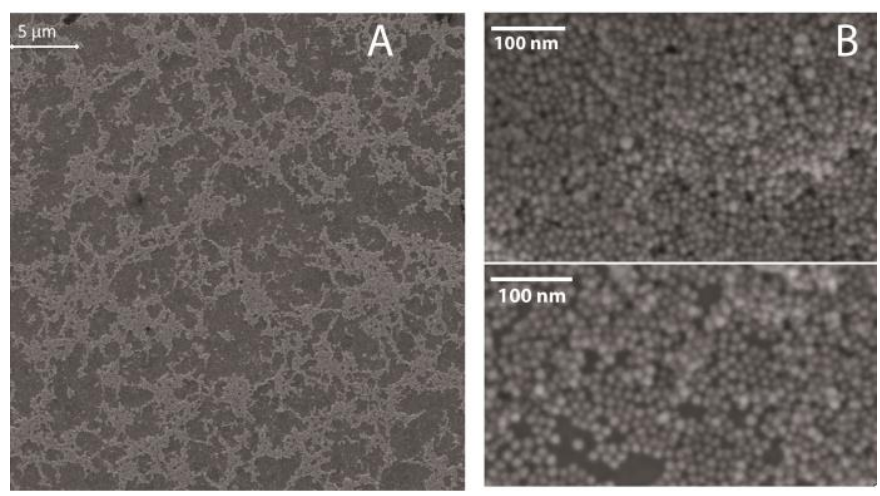

Fig. 6. A. SEM image of a large area $(30 \mu \mathrm{m} \times 30 \mu \mathrm{m})$ of gold surface covered by CTPR 20 and functionalized with GNPs. B. Representative SEM images of GNPs immobilized onto CTPR20 covering gold surface. Two areas in which is clear the close packed GNPs monolayer are displayed. SEM images are in the same scale as the AFM images of the protein monolayer displayed in Fig. 4. 